\title{
Enhanced metacognition for unexpected action outcomes
}

\author{
Daniel Yon ${ }^{1,2}$ \\ 1. Department of Psychology, Goldsmiths, University of London. \\ 2. Department of Psychological Sciences, Birkbeck, University of London \\ Correspondence: d.yon@gold.ac.uk
}

\section{Submitted to PsyArXiv on $16^{\text {th }}$ Dec 2020}

Metacognition allows us to explicitly represent the uncertainty in our perceptions and decisions. Recent theories suggest that we use predictive models of our environment to optimise these introspective processes, but extant accounts disagree about the role prediction plays: some accounts suggest that we should have more sensitive subjective insight for predictable events, while others stress that metacognition should be enhanced for surprising prediction errors. Here two experiments compare these accounts. Participants performed actions to generate visual outcomes that could move in expected or unexpected directions. Across both experiments, signal detection analyses revealed enhanced metacognition for unexpected outcomes. A combination of reverse correlation and computational modelling suggested this advantage arose because metacognitive processes are more sensitive to unexpected information. These results are consistent with higher order inference models of introspective awareness and point to a mechanism that may optimise diverse aspects of cognition and behaviour in an unstable world.

\section{Introduction}

Our minds are optimised by metacognition - the processes that allow us to monitor, represent and communicate properties of our own mental states ${ }^{1,2}$. For example, metacognitive processes allow us to generate explicit representations of confidence, which we can use to improve the accuracy of decisions and beliefs when we are uncertain ${ }^{3}$ : slowing down choices ${ }^{4}$, gathering more information ${ }^{5}$ or seeking advice from more certain others ${ }^{6,7}$.

However, while it is clear that metacognition optimises cognition and behaviour, it is less clear how metacognition is itself optimised. An influential idea across the mind sciences is that many aspects of cognition are finessed by predictive mechanisms which use probabilistic prior knowledge to shape perception, decision and belief ${ }^{8-10}$. Intriguingly, recent models have suggested that predictive knowledge may also be used to optimise metacognitive awareness - though different accounts have proposed prediction plays opposite and incompatible roles.

On the one hand, Bayesian models of metacognition suggest the top-down predictions enhance introspection about expected events ( ${ }^{11}$; see Fig. 1a). These accounts share a family resemblance to Bayesian models of perception ${ }^{9,12-14}$, which assume it is adaptive for sensory representations to be weighted towards predicted outcomes - making us more likely to see, hear or feel sensory events that we expect to occur ${ }^{15-18}$. Analogously to Bayesian of models of perception, Bayesian models of metacognition suggest that top-down predictions 'sharpen' internal representations of expected events, leading to more sensitive metacognition about predicted signals ${ }^{11,19}$. Consistent with this idea, studies have found observers have more reliable subjective insight about expected over unexpected events even when objective perceptual performance is matched ${ }^{11}$. Enhancing metacognition for expected events could be adaptive, as it will ensure agents have robust subjective representations of events that are - by definition - most likely to occur.

On the other hand, contrasting models suggest predictions are used to optimise metacognition in a fundamentally different way: by enhancing subjective awareness of prediction errors. For example, one recent 'higher order inference' model proposes a computational architecture where explicit awareness is the product of second-order metacognitive inferences about first-order perceptual representations ( ${ }^{20}$; see Fig. $1 \mathrm{~b}$ ). This framework assumes that each level of the cognitive hierarchy generates predictions about representations at lower levels and receives 'prediction error' signals reflecting the mismatch between expectations and reality 21,22 . This account bears a family resemblance to models of perceptual prediction which assume agents give relatively more weight to unexpected sensory signals - either by directly downweighting signals that are predictable 23,24 or by 
upweighting information that is especially surprising ${ }^{14}$. Indeed, this higher order inference model suggests that the same formal 'surprise' computation that we have suggested highlights unexpected events at the perceptual level (the Kullback-Leibler Divergence between hypotheses pre- and postinput ${ }^{14,25}$ ) is also implemented at higher hierarchical levels, which would ensure that unexpected events are more likely to enter metacognitive awareness 20 .

\section{a) Bayesian model}
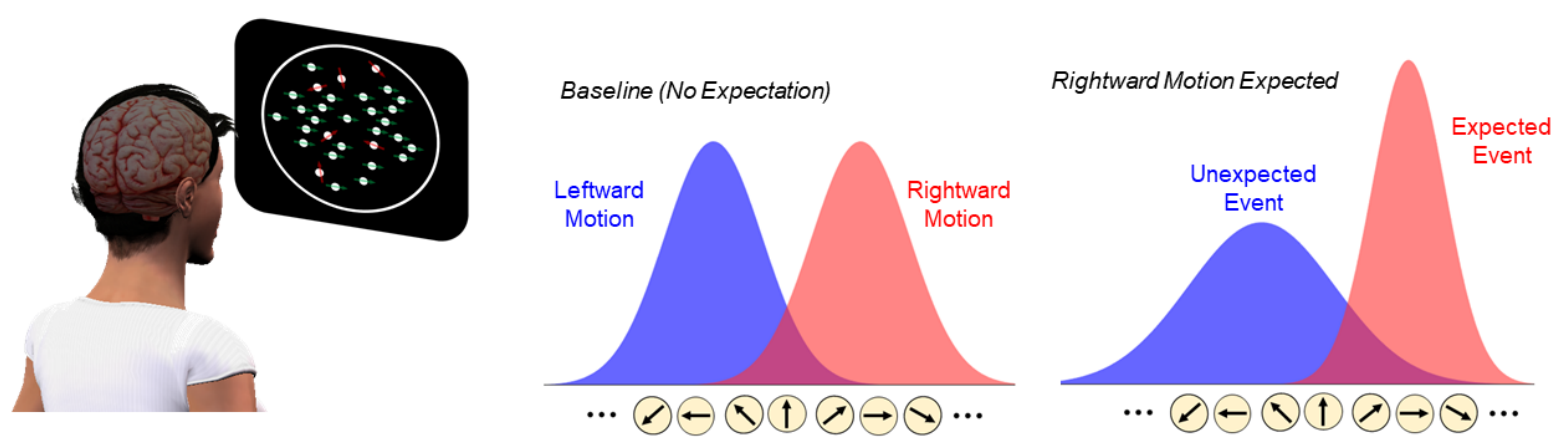

b) Higher-order inference model
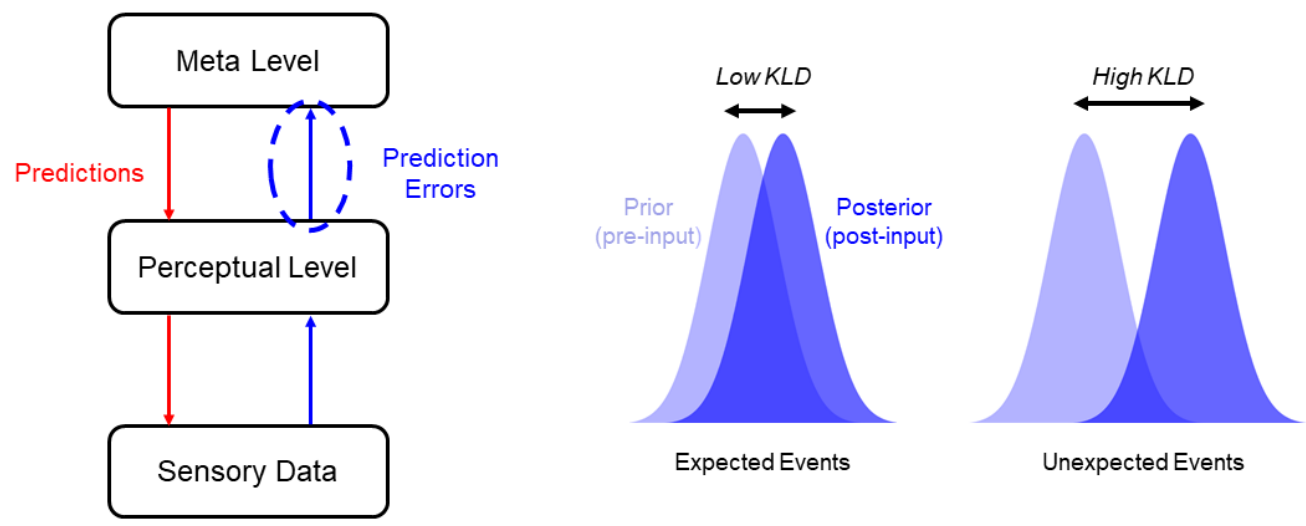

Figure 1 - Models of prediction and metacognition. (a) Bayesian models of perceptual metacognition suggest that we use top-down predictions to 'sharpen' representations of expected events. These theories suggest that increasing the gain on sensory units that code for expected features generates more precise internal representations for predicted events - leading to more sensitive metacognition about expected signals. (b) In contrast, higher-order inference models suggest that subjective introspection depends on prediction errors passed from perceptual to meta-level monitoring systems (left). In this architecture, access to meta-level awareness is gated by a computation of 'surprise' (Kullback-Leibler Divergence [KLD] right). Since KLD is higher for more surprising signals, these models suggest metacognition will be more sensitive for unexpected events.

While this contrasts with Bayesian models, sensitising metacognition to unexpected events could afford creatures particular adaptive advantages, given that prediction errors often signal the need to update our beliefs and adjust ongoing actions. Indeed, high fidelity metacognition about newsworthy errors would allow agents to coordinate other cognitive processes to rapidly adapt to surprises across modalities, given that explicit metacognition is thought to play a key role in broadcasting information across perceptual, attentional and motor systems ${ }^{26}$.

However, to date there has been no empirical evidence that metacognition of prediction errors is enhanced. This paper presents two experiments (one replication) that compared Bayesian and higher-order inference models of metacognition, investigating whether introspection is enhanced for expected or unexpected outcomes. Participants completed a combined action-perception task where they produced movements to generate moving dot stimuli yoked to their actions. Participants made perceptual decisions about the observed motion direction, while also indicating their level of 
confidence in their choices. Critically, stimuli could move in the same (expected) or opposite (unexpected) direction as the participant's action - allowing us to evaluate how metacognition changes as a function of expectations. To pre-empt the results, both experiments revealed enhanced metacognitive sensitivity for unexpected outcomes. Reverse correlation and computational modelling analyses suggested that such effects arose because metacognitive systems are particularly sensitive to unexpected signals. This combination of results is suggestive of a mechanism that optimises introspection about informative prediction errors, which may enhance cognition and behaviour in an unstable world.

\section{Results}

Separate groups of participants ( $N=24)$ in Experiments 1 and 2 completed an online psychophysical action-perception task (see Fig. 2). The procedure for both experiments was identical, but Experiment 2 acted as a preregistered replication (recorded on AsPredicted.org). On each trial, participants produced a left- or rightward movement with their computer mouse to generate a cloud of moving dots. While the displacement of every dot was yoked to the participant's own movement, a proportion of dots moved coherently left or right while the remaining dots moved in random directions. Importantly, on $50 \%$ of trials the dots were programmed to move in the expected direction (i.e., participant moves left, dots move left) or the unexpected direction (i.e., participant moves left, dots move right). To match objective discriminability separate adaptive staircases titrated stimulus strength across expected and unexpected conditions (see Methods). At the end of each trial, participants used a four-point scale to indicate whether the coherent dots moved left or right, alongside their decision confidence ('confident' or 'guess').

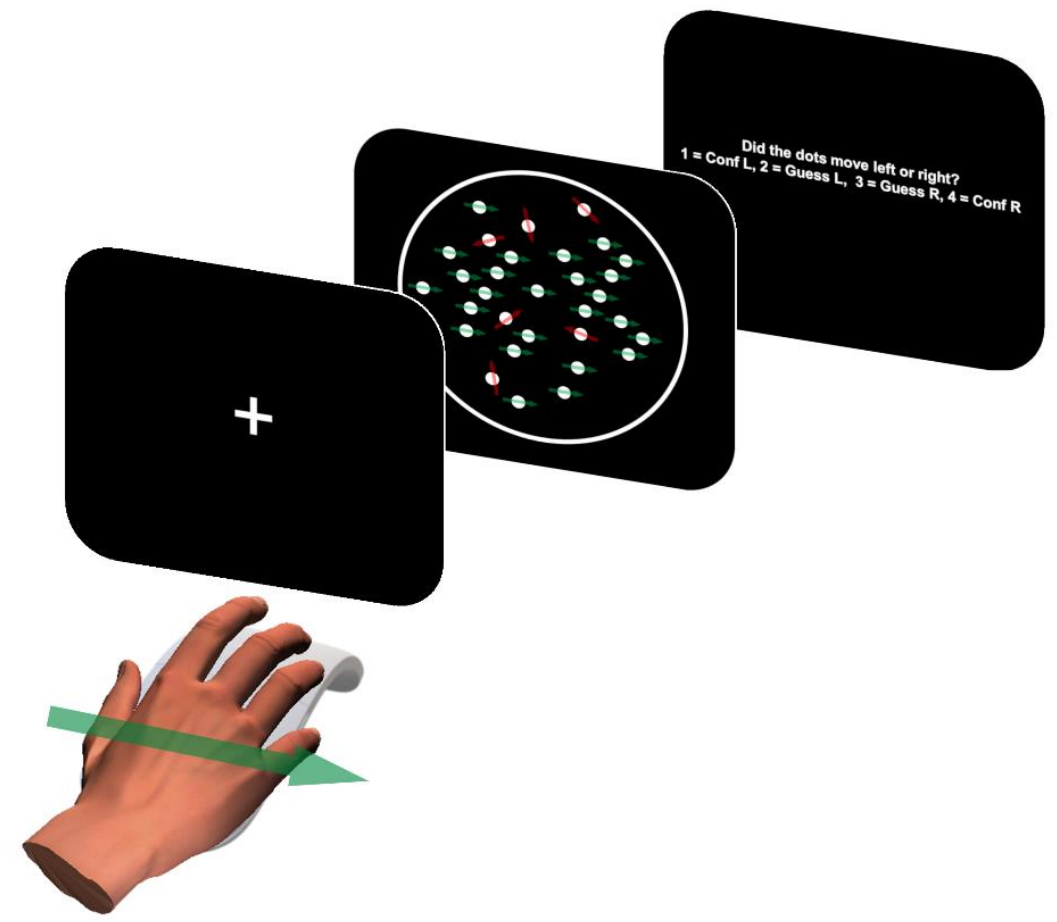

Figure 2: Metacognition about (un)expected action outcomes: Participants performed a task where their actions generated dot motion clouds that could move in expected or unexpected directions. At the end of each trial participants gave a combined perceptual decision (i.e., "did the dots move left or right?") and metacognitive report (i.e., "are you confident or guessing?").

\section{Enhanced metacognition for unexpected outcomes}

Separate measures of perceptual sensitivity (d') and metacognitive sensitivity (meta-d') were computed for expected and unexpected trials based on participant decisions. Higher perceptual sensitivity (d') indicates a better ability to objectively distinguish left- from right-moving motion, while higher metacognitive sensitivity (meta-d') indicates a closer coupling between subjective confidence and objective performance ${ }^{27-29}$. Analyses of these statistics revealed that objective discrimination 
performance (d') did not differ between expected and unexpected trials in Experiment 1 -mean (sem) expected $\mathrm{d}^{\prime}=1.38(.042)$, unexpected $\mathrm{d}^{\prime}=1.37(.052), t_{23}=.234, p=.817, d z=.048-$ or Experiment 2 - expected d' $=1.45$ (.098), unexpected d' $=1.44(.130), t_{23}=.209, p=.836, d z=.043$. However, equivalent analyses revealed enhanced metacognitive sensitivity (meta-d') on unexpected trials in Experiment $1-$ mean $\left(\right.$ sem) expected meta-d' $=.830(.128)$, unexpected meta-d' $=1.15(.111), t_{23}=$ 2.39, $p=.025, d z=.488$ - and Experiment 2 -expected meta-d' $=1.11$ (.201), unexpected meta-d'= $1.58(.177), t_{23}=2.81, p=.010, d z=.574$. This same pattern was revealed in the Mdiff measure of metacognitive efficiency (meta-d' $\left.-d^{\prime}\right)^{27}$, which was higher for unexpected than expected outcomes in both experiments $-t_{23}=2.29, p=.032, d z=.467(\operatorname{Exp} 1), t_{23}=2.34, p=.028, d z=.477(\operatorname{Exp} 2)$.

Critically, this suggests that agents have more sensitive introspection about prediction errors consistent with higher order inference models ${ }^{20}$ but contrary to Bayesian accounts ${ }^{11}$.

a) Enhanced metacognition (meta-d') for unexpected outcomes
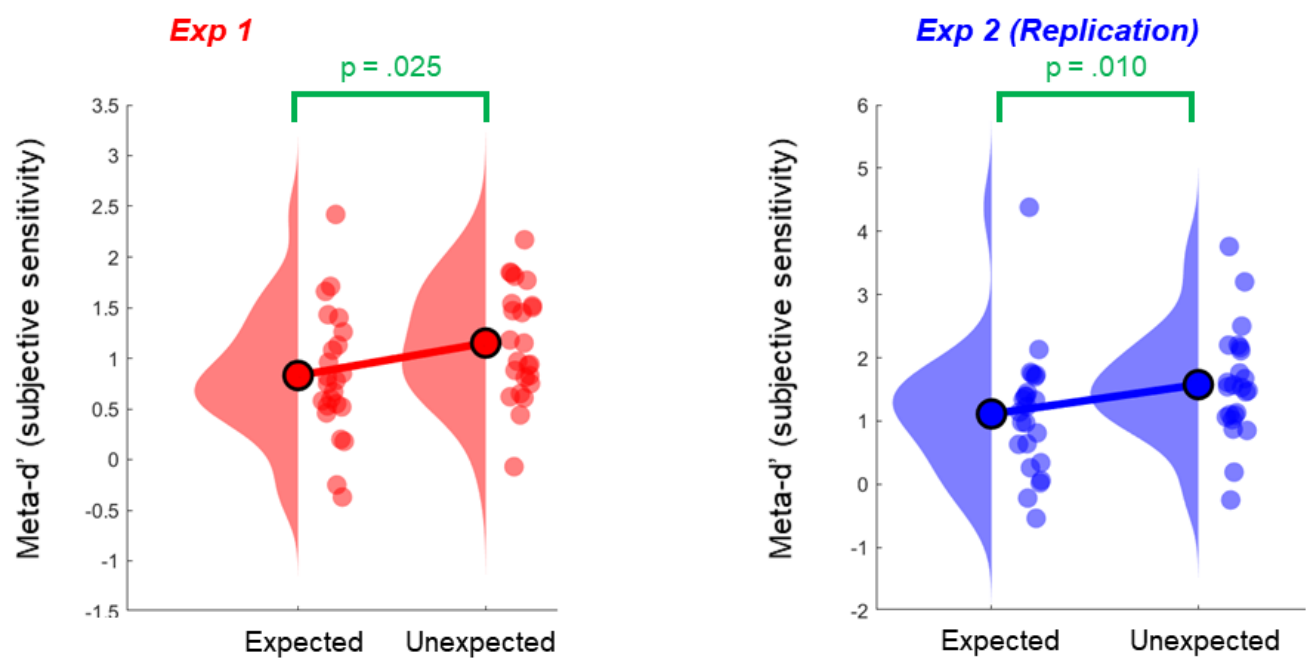

b) No difference in objective sensitivity (d')
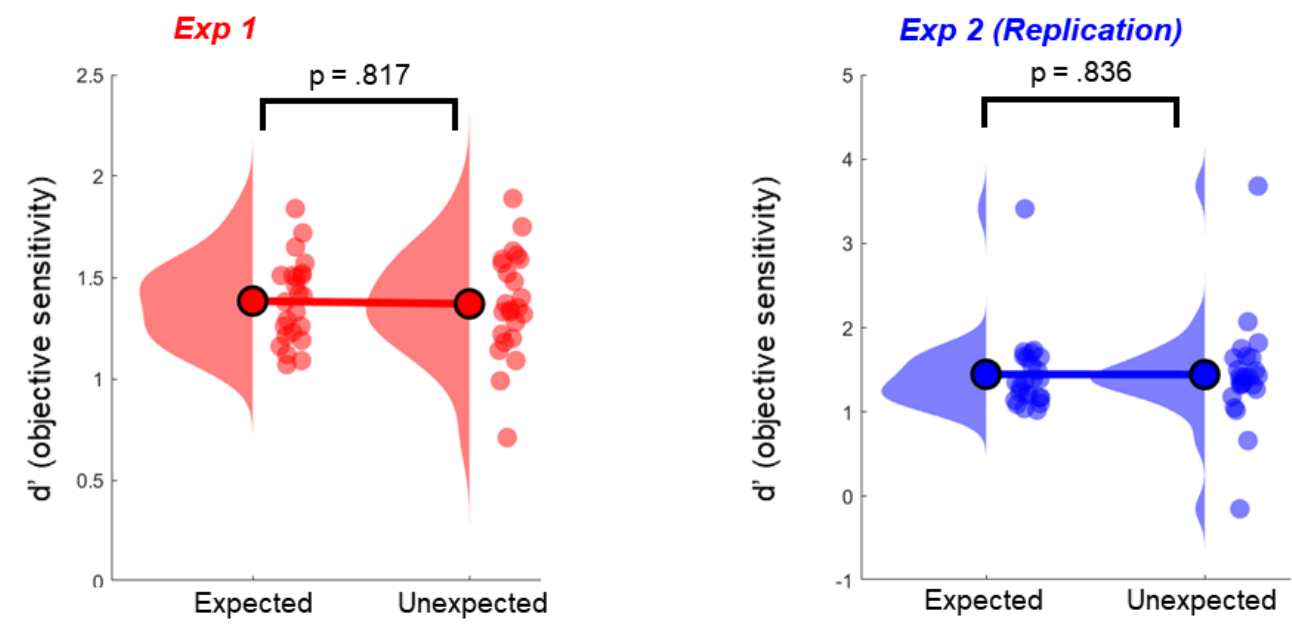

Figure 3: Measuring metacognitive sensitivity: Modelling signal detection theoretic measures to perceptual decisions revealed - across both experiments - that observers had more sensitive metacognition (higher metad') for unexpected outcomes (a). In other words, participants gave more accurate subjective reports (i.e. feeling confident when correct) about stimuli that violated predictions. Critically, there were no differences in observers' objective ability to discriminate expected or unexpected stimuli, indexed by d' (b) 
Reverse correlation and computational modelling reveal metacognitive decisions are more sensitive to unexpected signals

Signal detection analyses revealed that observers had more sensitive metacognition (higher meta-d') for unexpected action outcomes. Higher order inference models of awareness assume that metacognitive systems receive inputs from perceptual systems and suggest that such an effect could arise because metacognitive mechanisms afford more weight to unexpected information.

This possibility was tested in this paradigm using a combination of reverse correlation ${ }^{30}$ and computational modelling 17,31 to investigate how metacognitive decisions are coupled to motion information in observed stimuli, and whether this coupling is enhanced for signals that are unexpected. To this end, motion information was computed for each stimulus on each trial for each participant, determining how much the horizontal components of dot motion (both coherent and noise dots) matched the objectively programmed stimulus direction (see Methods). As illustrated in Fig 4a, random trial-by-trial noise sometimes spuriously resembled the programmed motion direction (creating more overall motion information) and sometimes spuriously opposed this direction (creating stimuli with less motion information).

These motion energy estimates were used to model trial-wise confidence reports and reaction times using the drift diffusion model. Sequential sampling models like the drift diffusion framework have enjoyed growing attention in the cognitive sciences ${ }^{32}$ and suggest that agents make two-choice decisions (e.g., "Am I confident or guessing?") by integrating evidence to a decision bound controlled by a drift rate $(v)$ parameter (see Fig $4 b$ ). Modelling our data in this way mirrors the information processing hierarchy hypothesised by higher order inference models of awareness and ('higher order thought' models more generally33), where metacognitive decisions depend on higher level integration of lower level perceptual signals ${ }^{20}$. Indeed, one can draw a formal equivalence between the drift rate $(v)$ in these models and the gain on information that ascends from lower to higher levels in hierarchical predictive loops ${ }^{34}$.

Drift diffusion models were fit to the combined data from Experiments 1 and 2, where metacognitive decisions were assumed to be coupled to trial-wise motion energy. This was achieved by estimating a parameter $v \sim$ Energy, which couples (metacognitive) evidence accumulation to motion energy: if this parameter is positive, observers will be more likely to report "confident" when motion energy is stronger and more likely to report "guess" when motion energy is weaker. In our model of interest two separate $v \sim$ Energy parameters were estimated for expected and unexpected trials, allowing us to investigate whether metacognition was more tightly coupled to expected or unexpected information. This was contrasted with a null model, which estimated a single $v \sim$ Energy parameter for both conditions.

These models were compared using deviance information criteria (DIC) as an approximation of Bayesian model evidence. Lower DIC indicates better model fit, accounting for model complexity. This comparison revealed that a model which allowed $v \sim$ Energy parameters to vary according to expectations provided a better fit than the null model ( $\triangle \mathrm{DIC}$ : -54.97). Inspecting group-level parameters in the winning model revealed the metacognitive decisions were more tightly coupled to unexpected signals (mean $v \sim$ Energy_Unexpected $=2.09$ ) than expected signals (mean $\checkmark \sim$ Energy_Expected $=1.57$; see Figure 4c). Moreover, correlating the subject-specific difference in $\checkmark \sim$ Energy parameters with differences in meta-d' (expected - unexpected) revealed strong relationships $-r_{48}=.462, p=.001$ (see Figure $4 d$.). These results support the idea that metacognition of unexpected events is enhanced because metacognitive mechanisms are especially sensitive to surprising signals (prediction errors) ${ }^{20}$. 
a)
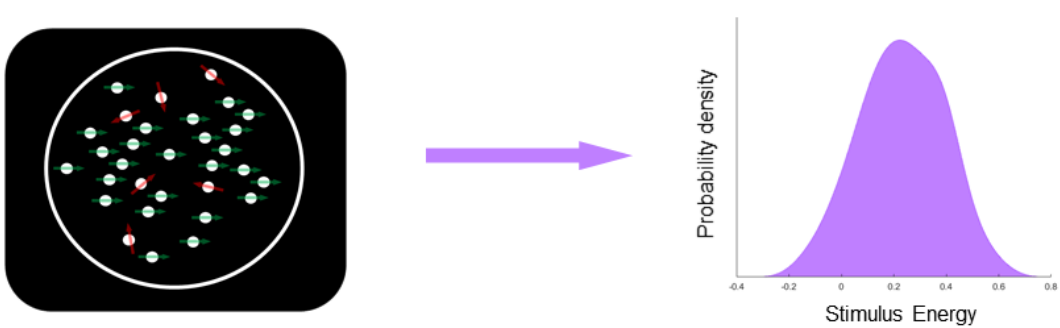

b)

Modelling metacognitive decisions
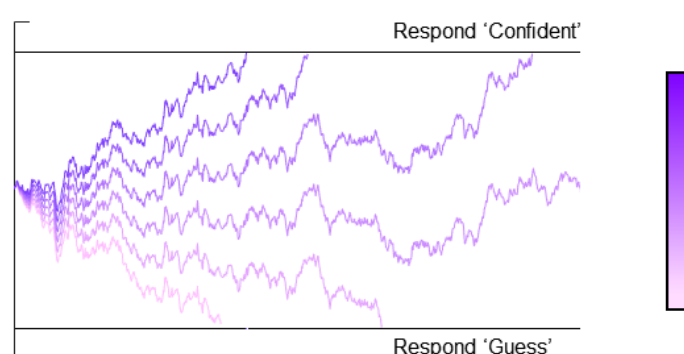

Stimulus Energy

(High to Low)
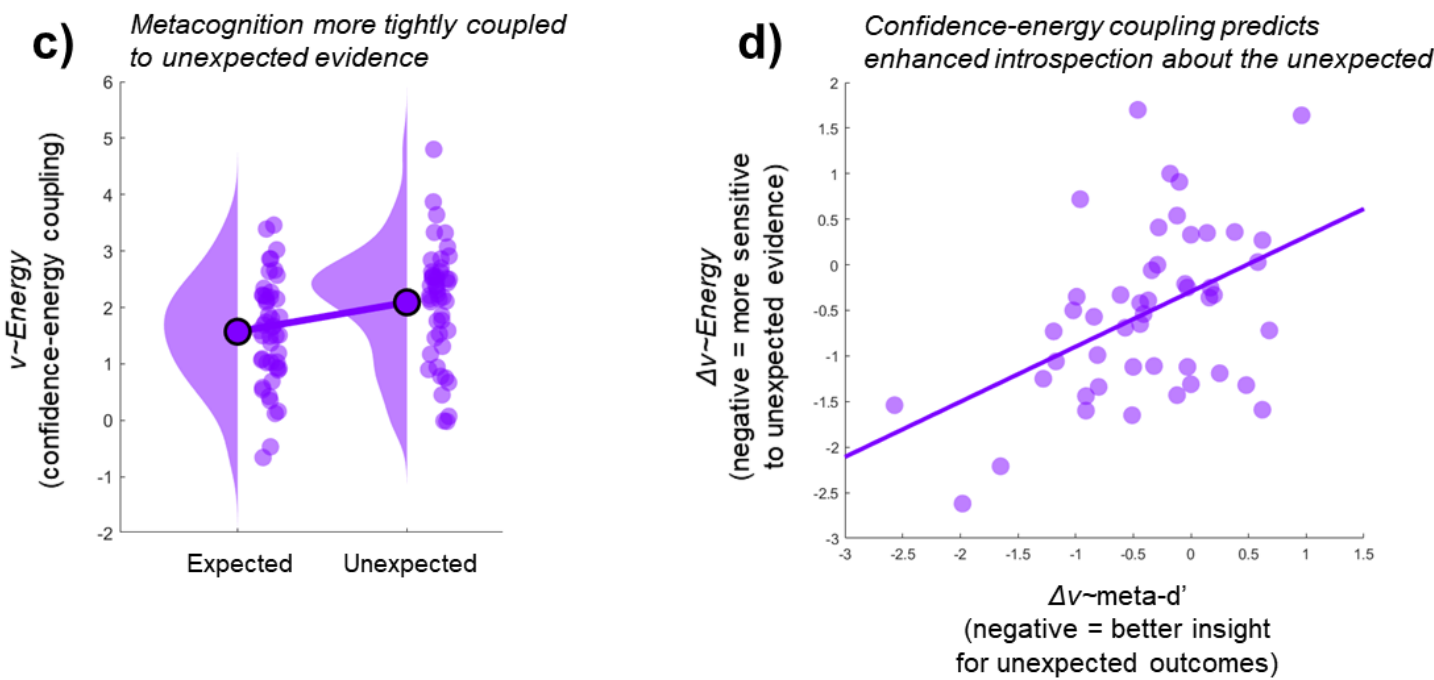

Figure 4. Modelling metacognitive decisions: To investigate how metacognitive mechanisms are coupled to expected and unexpected information we recorded the motion energy in each stimulus (a) and constructed drift diffusion models where signal strength on a given trial was used to predict confidence reports (b). This modelling procedure revealed that metacognitive evidence accumulation was more tightly coupled to unexpected signals (c) and variability in this model-based effect predicted empirical enhancements of metacognition for prediction errors (d).

\section{Discussion}

Existing models of metacognition disagree about how introspective processes should be shaped by predictions. Across two experiments we found that agents have more sensitive metacognition for unexpected action outcomes. Reverse correlation and computational modelling suggested that these effects arose because metacognitive mechanisms are especially sensitive to unexpected signals (i.e., prediction errors).

These findings support higher order inference models of metacognitive awareness, which assume that introspection depends on higher order mechanisms that receive inputs from lower levels in the 
cognitive hierarchy (e.g., from perceptual systems). Critically, access to these higher levels is assumed to depend on a 'surprise' computation (KLD' $\left.{ }^{14,25}\right)$, such that metacognition is enhanced for unexpected events (see Fig 1b). In line with this hypothesis, our results reveal that metacognitive mechanisms afford more weight to unexpected sensory signals, leading to higher fidelity metacognition about events that violate our initial predictions.

These results are hard to reconcile with Bayesian models, which assume metacognition is enhanced for expected outcomes. Such accounts have previously drawn support from studies showing superior perceptual metacognition when stimuli are predictably present or absent ${ }^{11}$ - an effect which may depend on the integration of top-down predictions and bottom-up evidence in particular frontal brain regions ${ }^{19}$. It is important to understand the discrepancy between these and present findings, especially since there are likely to be contexts where sensitising metacognition of expected events is functionally adaptive (e.g., in very stable environments where expectations are likely to come true).

Indeed, the tension between Bayesian and higher order inference models of metacognition is reminiscent of the tension between Bayesian and 'Cancellation' models of perceptual prediction, which respectively assume that expected signals are up- or down-weighted when making perceptual inferences. We have recently proposed a mechanistic model that has the potential to resolve this 'perceptual prediction paradox'14 - including via the idea that expected information will dominate perceptual inference when sensory signals are most uncertain, but surprising unexpected signals will be upweighted when sensory evidence is more precise. While the discrepancy between our present results and previous reports of Bayesian metacognition ${ }^{11}$ may point to a parallel 'metacognitive prediction paradox' it is not clear whether the same mechanistic ideas can solve this particular puzzle. For example, here we find enhanced metacognition for prediction errors, even though observers were generally very uncertain about the signals they were judging.

Enhanced metacognition for unexpected outcomes - as revealed in these experiments - could support cognition and behaviour in several ways. Detecting prediction errors is key to learning and action control - as unexpected events signal the need to update our beliefs and take new courses of action. For example, if we visit our favourite restaurant but are served a disgusting starter, we might revise our opinion about the skills of the chef and find somewhere else for dinner. Ensuring metacognitive awareness of such prediction errors could be adaptive over and above highlighting prediction errors in domain-specific networks, given that metacognitive mechanisms are well-poised to 'broadcast' unexpected information across different cognitive systems ${ }^{26}$. This would allow prediction errors detected in one modality or domain (e.g., perceptual or affective prediction errors associated with disgust) to effectively coordinate processing in others (e.g., memory systems to select a new restaurant, and motor systems to get your coat).

Enhanced subjective insight about prediction errors could also lead to more effective metacognitive control when we find ourselves in novel or surprising environments ${ }^{35}$. For example, more sensitive metacognition about prediction errors could support effective information-seeking behaviour to resolve uncertainty about the causes of unexpected environmental changes ${ }^{5}$. Moreover, it has been suggested that explicit metacognition plays an important social function - allowing us to share mental states with others ${ }^{36}$. Surprise-induced enhancements of metacognition within individual minds may therefore support groups as they make sense of unexpected changes together, since sharing information with more reliable expressions of confidence will lead to a more accurate consensus about what has occurred and why 6,37 .

In conclusion, these data show that agents have enhanced metacognition for unexpected action outcomes. Computational modelling revealed this introspective advantage for prediction errors arises because metacognitive systems are especially sensitive to surprising signals. While inconsistent with Bayesian models of metacognition, these findings accord well with higher order inference accounts, and are suggestive of a mechanism that optimises introspection in an unstable world.

\section{Methods}

Participants: Two independent samples $(N=24)$ took part in Experiments 1 and 2, recruited via the online testing platform Prolific. This sample size was chosen based on pilot testing, which found an effect of expectations on confidence $d z=.573$. A sample of 24 provides $>85 \%$ power to detect effects at least this large. Some participants 
in each experiment (eleven in Experiment 1, six in Experiment 2) were excluded from the final sample for failing to follow task instructions (i.e. not executing movements as instructed on $\geq 200$ trials). This exclusion rate is typical for online experiments ${ }^{38}$ and this exclusion criterion was pre-registered for Experiment 2 (https://aspredicted.org/w7hg2.pdf).

Participants were pre-screened to ensure they were right-handed, aged 18-50 (mean (sd) age in Experiment 1 27.1 (9.2) years - and Experiment 2 - 29.7 (8.7) years), native English speakers resident in the United Kingdom, with normal (or corrected-to-normal) vision and no history of mental illness. Thirteen men and 11 women took part in Experiment 1, and 14 men and 10 women took part in Experiment 2. Participants were paid a small honorarium via Prolific for taking part. All procedures were approved by the ethics committee at Goldsmiths, University of London and complied with the Declaration of Helsinki.

Experimental task: Participants completed an action-perception metacognition task programmed in PsychoPy ${ }^{39}$. At the beginning of each trial, participants moved a white dot cursor into a central starting zone. Once in the zone for $2000 \mathrm{~ms}$, a white fixation cross appeared - indicating that participants were free to execute either a left or rightwards mouse movement. Participant movements generated a dot motion cloud, where the displacement of each dot was yoked frame-by-frame to the horizontal displacement recorded in the participant's action. A proportion of coherent dots moved either leftwards or rightwards on every trial, while the remaining dots moved in random directions. Motion stimuli were presented for $300 \mathrm{~ms}$, followed by a question screen where participants indicated on a four point scale the direction of motion and their confidence in their decision ( 1 = Confident Left, 2 = Guess Left, 3 = Guess Right, 4 = Confident Right).

Critically, $50 \%$ of trials had expected stimuli - where coherent dots moved in the same direction as the participant's action (e.g., move left, see left) - while $50 \%$ of trials had unexpected stimuli - where coherent dots moved in the opposite direction to the executed action (e.g., move left, see right). This allows us to measure how metacognition varied as a function of expectations, since although expected and unexpected trials are equiprobable within the task, participants have learned over the course of decades (and perhaps 'hardwired') the prediction that hand movements generate congruent visual motion ${ }^{40-42}$. Since previous studies have shown that action can enhance ${ }^{17,43}$ or attenuate ${ }^{44,45}$ perception of expected visual outcomes, separate adaptive staircases were used to titrate stimulus strength on expected and unexpected trials to ensure that any differences in metacognition were not confounded with differences in objective perceptual accuracy ${ }^{27}$. Both staircases adjusted stimulus strength according to a 1-up-1-down rule starting at 40\% coherence and reducing coherence after each correct decision (to a minimum of $4 \%$ ) and increasing coherence after each incorrect decision (to a maximum of $80 \%$ ), with a fixed step size of $+/-4 \%$. Such a staircase targets the lowest coherence level where an individual observer can distinguish left- from right-moving motion. Objective signal strength was well-matched across expected and unexpected trials, with a mean (sd) coherence of $25.1(12.1) \%$ and $27.6(14.5) \%$ respectively.

Participants completed a short practice block (20 trials) where they received feedback about decision accuracy before the main experiment. The main experiment comprised 240 trials, no decision feedback was given and breaks were taken every 20 trials.

Data screening: Data from individual trials were excluded from further analyses where recorded motion traces revealed participants had not moved in a single, horizontal direction (left or right), generating impossible-to-judge stimuli (e.g., dot motion clouds that coherently moved left and right). Participants were excluded from analyses entirely if their datasets contained fewer than 200 valid trials, as small trial numbers reduce the sensitivity of subsequent analyses (see below) and suggest an inability to follow task instructions. This criterion was applied to both experiments and preregistered for Experiment 2.

Signal detection measures: Objective sensitivity and metacognitive sensitivity was indexed by computing the statistics d' and meta-d'. In this task, d' reflects an observer's objective ability to distinguish left- from right-moving dot motions ${ }^{46}$. In contrast, meta-d' is an analogous statistic reflecting how accurately subjective confidence ratings ('confident' or 'guess') track decision accuracy ${ }^{28}$. These statistics were modelled simultaneously to participant decisions using the non-hierarchical variant of the hMeta-d' Bayesian estimation procedure developed by Fleming ${ }^{29}$. This approach uses Monte Carlo Markov Chain (MCMC) sampling to estimate best fitting parameter values for individual subjects in each condition. These analyses used three chains of 5000 samples (20\% 'burn-in'), and model convergence was assessed by calculating Gelman-Rubin statistics (all $\hat{r}<1.1$ ).

Reverse correlation and computational modelling: To investigate the coupling between metacognitive decisions and expected and unexpected signals, we computed the motion information in each stimulus on each trial. This involved calculating the horizontal motion component of each moving dot (given by the cosine of the motion angle) and averaging these motion components across all dots in the display. This generated an estimate of stimulus energy theoretically bounded between 1 (all dots move in the coherent direction) and -1 (all dots move opposite to the coherent direction). Note that this calculation is symmetric for both stimulus directions i.e. higher 
numbers indicate stronger leftward motion in left-moving stimuli and stronger rightward motion in right-moving stimuli).

These trial-wise estimates of stimulus energy were combined with drift diffusion modelling of metacognitive choices, to establish whether metacognition is more sensitive to unexpected information. Models were fit using the hddm package developed by Wiecki et al ${ }^{31}$, which uses a hierarchical Bayesian modelling approach to simultaneously estimate group-level and subject-specific parameters based on choices ('confident' vs 'guess') and reaction times. Modelling analyses were limited to those trials where objective perceptual decisions (left vs right) were correct (to avoid introducing a circularity between model-based and signal detection measures) and where reaction time was $>100 \mathrm{~ms}$ (as shorter responses are likely to be anticipations, and implausibly short reaction times cannot be modelled by the DDM). Parameters were estimated using MCMC sampling, with 10,000 samples (25\% burn-in), convergence was assessed by inspecting chain posteriors and models were compared using deviance information criteria as an approximation of Bayesian model evidence.

Statistical information: All statistical tests were two-tailed, using alpha $=.05$. For all statistical comparisons, outliers on effects (absolute $z$ score $>2.5$ ) were winsorized to $z=2.5$ for inferential tests. This aspect of the analysis was pre-registered and was applied to one outlier each on the expected - unexpected meta- $d$ ' and expected - unexpected Mdiff comparison in both Experiment 1 and 2, as well as one outlier on the d' analysis in Experiment 2. While inferential statistics after winsorization are reported in the Results above, data patterns (and their statistical significance) were unchanged by these adjustments.

\section{References}

1. Flavell, J. H. Metacognition and cognitive monitoring: A new area of cognitive-developmental inquiry. American Psychologist 34, 906-911 (1979).

2. Fleming, S. M., Dolan, R. J. \& Frith, C. D. Metacognition: computation, biology and function. Philos. Trans. R. Soc. Lond., B, Biol. Sci. 367, 1280-1286 (2012).

3. Gilbert, S. J. et al. Optimal use of reminders: Metacognition, effort, and cognitive offloading. Journal of Experimental Psychology: General 149, 501-517 (2020).

4. Yeung, N. \& Summerfield, C. Metacognition in human decision-making: confidence and error monitoring. Philosophical Transactions of the Royal Society B: Biological Sciences 367, 1310-1321 (2012).

5. Desender, K., Boldt, A. \& Yeung, N. Subjective confidence predicts information seeking in decision making. Psychol Sci 29, 761-778 (2018).

6. Bahrami, B. et al. Optimally interacting minds. Science 329, 1081-1085 (2010).

7. Shea, N. et al. Supra-personal cognitive control and metacognition. Trends Cogn Sci 18, 186-193 (2014).

8. Clark, A. Whatever next? Predictive brains, situated agents, and the future of cognitive science. Behav Brain Sci 36, 181-204 (2013).

9. Yuille, A. \& Kersten, D. Vision as Bayesian inference: analysis by synthesis? Trends Cogn. Sci. (Regul. Ed.) 10, 301-308 (2006).

10. Körding, K. P. \& Wolpert, D. M. Bayesian decision theory in sensorimotor control. Trends in Cognitive Sciences 10, 319-326 (2006).

11. Sherman, M. T., Seth, A. K., Barrett, A. B. \& Kanai, R. Prior expectations facilitate metacognition for perceptual decision. Conscious Cogn 35, 53-65 (2015).

12. Lange, F. P. de, Heilbron, M. \& Kok, P. How do expectations shape perception? Trends in Cognitive Sciences 22, 764-779 (2018).

13. Press, C. \& Yon, D. Perceptual prediction: Rapidly making sense of a noisy world. Current Biology 29, R751-R753 (2019).

14. Press, C., Kok, P. \& Yon, D. The perceptual prediction paradox. Trends in Cognitive Sciences 24, 13-24 (2020).

15. Teufel, C., Dakin, S. C. \& Fletcher, P. C. Prior object-knowledge sharpens properties of early visual featuredetectors. Sci Rep 8, 10853 (2018).

16. Powers, A. R., Mathys, C. \& Corlett, P. R. Pavlovian conditioning-induced hallucinations result from overweighting of perceptual priors. Science 357, 596-600 (2017).

17. Yon, D., Zainzinger, V., de Lange, F. P., Eimer, M. \& Press, C. Action biases perceptual decisions toward expected outcomes. J Exp Psychol Gen (2020) doi:10.1037/xge0000826.

18. Thomas, E. R., Yon, D., Lange, F. P. de \& Press, C. Action enhances predicted touch. bioRxiv 2020.03.26.007559 (2020) doi:10.1101/2020.03.26.007559.

19. Sherman, M. T., Seth, A. K. \& Kanai, R. Predictions shape confidence in right inferior frontal gyrus. J. Neurosci. 36, 10323-10336 (2016).

20. Fleming, S. M. Awareness as inference in a higher-order state space. Neurosci Conscious 2020, (2020).

21. Rao, R. P. \& Ballard, D. H. Predictive coding in the visual cortex: a functional interpretation of some extraclassical receptive-field effects. Nat. Neurosci. 2, 79-87 (1999).

22. Friston, K. The free-energy principle: a unified brain theory? Nat. Rev. Neurosci. 11, 127-138 (2010).

23. Bays, P. M. \& Wolpert, D. M. Computational principles of sensorimotor control that minimize uncertainty and variability. J. Physiol. (Lond.) 578, 387-396 (2007).

24. Richter, D. \& de Lange, F. P. Statistical learning attenuates visual activity only for attended stimuli. eLife 8,. 
25. Itti, L. \& Baldi, P. Bayesian surprise attracts human attention. Vision Research 49, 1295-1306 (2009).

26. Shea, N. \& Frith, C. D. The global workspace needs metacognition. Trends in Cognitive Sciences 23, 560571 (2019).

27. Fleming, S. M. \& Lau, H. C. How to measure metacognition. Front. Hum. Neurosci. 8, (2014).

28. Maniscalco, B. \& Lau, H. A signal detection theoretic approach for estimating metacognitive sensitivity from confidence ratings. Conscious Cogn 21, 422-430 (2012).

29. Fleming, S. M. HMeta-d: hierarchical Bayesian estimation of metacognitive efficiency from confidence ratings. Neurosci Conscious 2017, (2017).

30. Wyart, V., Nobre, A. C. \& Summerfield, C. Dissociable prior influences of signal probability and relevance on visual contrast sensitivity. Proc. Natl. Acad. Sci. U.S.A. 109, 3593-3598 (2012).

31. Wiecki, T. V., Sofer, I. \& Frank, M. J. HDDM: Hierarchical Bayesian estimation of the drift-diffusion model in Python. Front Neuroinform 7, 14 (2013).

32. Ratcliff, R., Smith, P. L., Brown, S. D. \& McKoon, G. Diffusion decision model: current issues and history. Trends Cogn Sci 20, 260-281 (2016).

33. Lau, H. \& Rosenthal, D. Empirical support for higher-order theories of conscious awareness. Trends Cogn. Sci. 15, 365-373 (2011).

34. Limongi, R., Bohaterewicz, B., Nowicka, M., Plewka, A. \& Friston, K. J. Knowing when to stop: Aberrant precision and evidence accumulation in schizophrenia. Schizophrenia Research 197, 386-391 (2018).

35. Nelson, T. O. \& Narens, L. Why investigate metacognition? in Metacognition: Knowing about knowing 1-25 (The MIT Press, 1994)

36. Frith, C. D. The role of metacognition in human social interactions. Philos Trans $R$ Soc Lond B Biol Sci 367 , 2213-2223 (2012).

37. Bang, D. et al. Confidence matching in group decision-making. Nature Human Behaviour 1, 1-7 (2017).

38. Chandler, J., Mueller, P. \& Paolacci, G. Nonnaïveté among Amazon Mechanical Turk workers: Consequences and solutions for behavioral researchers. Behav Res 46, 112-130 (2014).

39. Peirce, J. et al. PsychoPy2: Experiments in behavior made easy. Behav Res 51, 195-203 (2019).

40. Dogge, M., Custers, R., Gayet, S., Hoijtink, H. \& Aarts, H. Perception of action-outcomes is shaped by lifelong and contextual expectations. Scientific Reports 9, 5225 (2019).

41. Hommel, B., Müsseler, J., Aschersleben, G. \& Prinz, W. The Theory of Event Coding (TEC): a framework for perception and action planning. Behav Brain Sci 24, 849-878; discussion 878-937 (2001).

42. Yon, D., de Lange, F. P. \& Press, C. The predictive brain as a stubborn scientist. Trends Cogn. Sci. 23, 6-8 (2019).

43. Christensen, A., Ilg, W. \& Giese, M. A. Spatiotemporal tuning of the facilitation of biological motion perception by concurrent motor execution. J. Neurosci. 31, 3493-3499 (2011).

44. Cardoso-Leite, P., Mamassian, P., Schütz-Bosbach, S. \& Waszak, F. A new look at sensory attenuation: Action-effect anticipation affects sensitivity, not response bias. Psychol Sci 21, 1740-1745 (2010).

45. Lally, N., Frendo, B. \& Diedrichsen, J. Sensory cancellation of self-movement facilitates visual motion detection. J Vis 11, (2011).

46. Green, D. M. \& Swets, J. A. Signal detection theory and psychophysics. (John Wiley, 1966).

\section{Acknowledgements}

I am grateful to Clare Press for comments on an earlier draft of this manuscript, and to members of the Institute of Philosophy Lab Meeting for helpful discussions. 